\title{
In silico Assessment of Antiarrhythmic Effects of Drug Ranolazine on Electrical Activity in Human Ventricular Myocardium
}

\author{
Mitra Abbasi ${ }^{1}$, Sebastian Polak ${ }^{1,2}$ \\ ${ }^{1}$ Simcyp Limited (a Certara Company), Blades Enterprise Centre, Sheffield, UK \\ ${ }^{2}$ Faculty of Pharmacy, Jagiellonian University Medical College, Krakow, Poland
}

\begin{abstract}
The aim of this study was to simulate the electrophysiological modifications by the ranolazine at the left ventricular wall level by using in vitro electrophysiological measurements from canine ventricular myocytes to generate early information on cardiac safety in the human heart. The antiarrhythmic effect of ranolazine on two proarrhythmic drugs was examined.

The sensitivity of ionic currents to ranolazine was described as Action Potential Duration (APD) variations in response to different therapeutic concentrations. The observed results can be explained by the interplay between inhibition of the $I_{N a L}$ and $I_{K r}$ currents. Simulation results are in agreement with in vitro and in vivo studies of arrhythmia and confirmed the antiarrhythmic properties of ranolazine which may be utilized for suppressing ventricular arrhythmias.
\end{abstract}

\section{Introduction}

Ranolazine is an antianginal compound, approved by the US Food and Drug Adminstration (FDA) [1,2] without significant hemodynamic fluctuations (e.g bradycardia or hypotension). However, ranolazine has been known to produce a small prolongation of the QT interval [1]. Some experimental evidences [2-5] indicated the ant-ischaemic effects of ranolazine which relate to inhibition of the late inward sodium current $\left(I_{N a L}\right)$ during cardiac repolarisation. Although several experimental studies [5-7] have shown a potent of antarrhythmic effect of ranolazine against cardiac (atrial and ventricular) arrhythmias, the potential antiarrhythmic effects of ranolazine need to be investigated in humans.

This study designed to evaluate the potential antiarrhythmic actions of ranolazine. For this purpose, a combination of ranolazine with class III antiarrhythmic drugs (dofetilide and d,l-sotalol) is used to examine the effects of inhibition of $I_{N a L}$ on top of $I_{K r}$ block. The current study demonstrates the impact of these drugs on APD prolongation and the underlying electrophysiological effect of ranolazine in a human ventricular myocytes cell model [8].

\section{Methods}

This study was designed to provide safety pharmacology modelling and simulation approaches to develop an in-silico tool for preclinical antiarrhythmic drug safety assessment by demonstrating the impact of dose/concentation relationship of drugs on ventricular arrhythmias biomarkers (i.e. APD, $\triangle \mathrm{APD}$ ). We obtained $\triangle \mathrm{APD}$ values $((\triangle \mathrm{APD}=\mathrm{APD}$ with ionic current inhibition (Table 1,2 and 3) - APD placebo (under control conditions)) from simulation models using experimental values from $[9,10]$.

We used the Cardiac Safety Simulator (CSS V2.0, Simcyp, Sheffield) to evaluate the cardiac electrophysiological effects of ranolazine and its interaction with two class III antiarrhythmic drugs.

\subsection{Model input data}

We used previously published data [9], as presented in Table 1, where half maximal inhibitory concentrations $\left(I_{C 50}\right)$ of ranolazine for five different ionic channels (i.e. the rapid delayed rectifier potassium current $\left(I_{K r} / \mathrm{hERG}\right)$, sodium current ( $\left.I_{N a} / \mathrm{Nav1.5}\right)$, late inward sodium current $\left(I_{N a L}\right)$, L-type calcium current $\left(I_{C a} /\right.$ Cav1.2), slow delayed rectifier current $\left(I_{K S} / \mathrm{KCNQ} 1+\mathrm{KCNE} 1\right)$ with $\mathrm{h}$ to describe the drug triggered ionic current modifications and the drug concentration $(\mu \mathrm{M})$.

We used drug concentrations and inhibitory actions of dofetilide and d,l-sotalol on ion channels in vitro from previously published data [10] as presented in Tables 2 and 3. Inhibitory actions (i.e. the dose-inhibition relationship) of these two compounds were specified at ion channels by using multiples of the free effective therapeutic plasma concentration $\left(E T P C_{\text {unbound }}\right)$ of the drug at 1, 10, 30, 50, 75 and 100 fold of their respective 
$E T P C_{\text {unbound }}$ value (Tables 2 and 3). We scaled from the original values obtained on $\mathrm{CHO}$ cells at room temperature to HEK cells at physiological temperature as the standard in vitro system for each set of data. Simulations were performed by using data presented in Table 4 , in order to test the potent late sodium $\left(I_{N a L}\right)$ blocking actions of ranolazine on suppressing arrhythmias induced by dofetilide and d,l-sotalol at different concentration.

Table 1. Drug concentrations and inhibitory actions of ranolazine on ion channels in vitro

\begin{tabular}{|c|c|c|c|c|c|c|}
\hline \multicolumn{7}{|c|}{$\begin{array}{l}\text { Effects of ranolazine on cardiac transmembrane ion } \\
\text { currents [9] }\end{array}$} \\
\hline \multicolumn{2}{|c|}{ Ion Channel current } & \multicolumn{5}{|c|}{$\begin{array}{c}\text { Inhibitory Potency } \\
I_{C 50}(\mu \mathrm{M})\end{array}$} \\
\hline \multirow{3}{*}{ Inward } & $I_{N a}$ & \multicolumn{5}{|c|}{294} \\
\hline & $I_{N a L}$ & \multicolumn{5}{|c|}{5.9} \\
\hline & $I_{C a L}$ & \multicolumn{5}{|c|}{296} \\
\hline \multirow{4}{*}{ Outward } & $I_{K r}$ & \multicolumn{5}{|c|}{11.5} \\
\hline & $I_{K s}$ & \multicolumn{5}{|c|}{30} \\
\hline & $I_{K 1}$ & \multicolumn{5}{|c|}{ no effect } \\
\hline & $I_{\text {to }}$ & \multicolumn{5}{|c|}{ no effect } \\
\hline $\begin{array}{c}\text { Drug } \\
\text { concentrat } \\
\text { ion }(\mu \mathrm{M})\end{array}$ & Placebo & 2 & 4 & 5 & 10 & $15^{*}$ \\
\hline
\end{tabular}

* We assessed the drug actions at concentrations exceeding the plasma levels found in clinical trials $(>10$ $\mu \mathrm{M})[11]$.

Table 2. Drug concentrations and inhibitory actions of dofetilide on ion channels in vitro

\begin{tabular}{|c|c|c|c|c|c|c|c|}
\hline \multicolumn{8}{|c|}{ Inhibitory actions of dofetilide on ion channels [10] } \\
\hline \multirow{2}{*}{$\begin{array}{c}\text { Fracti } \\
\text { on } \\
\text { conc. }\end{array}$} & \multirow{2}{*}{$\begin{array}{c}E T P C_{\text {unbou }} \\
(\mu \mathrm{m})\end{array}$} & \multicolumn{2}{|c|}{$I_{N a}$} & \multicolumn{2}{|c|}{$I_{C a}$} & \multicolumn{2}{|c|}{$I_{K r}$} \\
\hline & & $I_{C 50}$ & h & $I_{C 50}$ & h & $I_{C 50}$ & h \\
\hline$\times 1$ & 0.0016 & \multirow{5}{*}{$\begin{array}{c}124 . \\
45\end{array}$} & \multirow{5}{*}{$\begin{array}{c}0.3 \\
2\end{array}$} & \multirow{5}{*}{$\begin{array}{c}184 \\
.1\end{array}$} & \multirow{5}{*}{$\begin{array}{c}0.8 \\
9\end{array}$} & \multirow{5}{*}{$\begin{array}{l}0.0 \\
38\end{array}$} & \multirow{5}{*}{$\begin{array}{c}1.9 \\
8\end{array}$} \\
\hline$\times 10$ & 0.016 & & & & & & \\
\hline$\times 30$ & 0.048 & & & & & & \\
\hline$\times 50$ & 0.08 & & & & & & \\
\hline$\times 75$ & 0.12 & & & & & & \\
\hline
\end{tabular}

Table 3. Drug concentrations and inhibitory actions of d,1-sotalol on ion channels in vitro.

Inhibitory actions of d,l-sotalol on ion channels [10]

\begin{tabular}{|c|c|c|c|}
\hline \multicolumn{2}{|c|}{ Inhibitory actions of d,l-sotalol on ion channels [10] } \\
\hline \multirow{2}{*}{ Fraction conc. } & \multirow{2}{*}{$E T P C_{\text {unbound }}$} & \multicolumn{2}{|c|}{$I_{K r}$} \\
& & $I_{C 50}$ & $\mathrm{~h}$ \\
\hline$\times 1$ & 14.68 & & \\
\hline$\times 10$ & 146.8 & \multirow{3}{*}{356.4} & \multirow{2}{*}{1.023} \\
\hline$\times 50$ & 734 & & \\
\hline$\times 75$ & 1101 & & \\
\hline$\times 100$ & 1468 & \\
\hline
\end{tabular}

Table 4. Drug concentrations data to assess drug-drug interactions of ranolazine with two unsafe compounds

\begin{tabular}{|c|c|c|c|c|c|}
\hline \multicolumn{6}{|c|}{ Ranolazine effects on dofetilide and d,1-sotalol } \\
\hline Substrate & \multicolumn{5}{|c|}{15} \\
\hline ranolazine & \multicolumn{5}{|c|}{ Drug concentration $(\mu \mathrm{M})$} \\
\hline dofetilide & 0.0016 & 0.016 & 0.048 & 0.08 & 0.12 \\
\hline d,l-sotalol & 14.68 & 146.8 & 734 & 1101 & 1468 \\
\hline
\end{tabular}

\subsection{Human ventricular cell model}

The computational models for endocardial (endo) cell type were derived from the O'Hara-Rudy dynamic (ORd) human ventricular model [8] by proportionally changing ionic currents based on in vitro measurements.

\subsection{Cardiac Safety Simulator (CSS)}

To assess the antiarrhythmic potency of the ranolazine and its interactions with the other two compounds, the CSS platform (V2.0) was used to simulate normal (control) physiological and drug-induced pathophysiological alterations in cardiac AP conduction at the population level (i.e. Sim-NEurCaucasian, North European Caucasian) [12]. The variability in the Sarcoplasmic reticulum volume was set to $30 \% \mathrm{CV}$ providing an estimate of the scale of variation.

\section{Results}

\subsection{Electrophysiological effects}

The effect of ranolazine on the AP of human ventricular endo cells is shown in Figure 1. Results show ranolazine has no effect on resting membrane potential and this is because of the lack of effect of the compound on the inward rectifier potassium channel current $\left(I_{K 1}\right)$. This drug has no influence on AP amplitude in ventricular myocytes. Ranolazine caused a small concentrationdependent lengthening of APD in endo cell.

Figures 2, 3 and 4 illustrate changes in APD ( $\triangle \mathrm{APD})$ for ranolazine, d,l-sotalol and dofetilide respectively. The $\triangle \mathrm{APD}$ increasingly grows by increasing dose for each compound. The concentration-dependent prolongation of APD that was greater at $90 \%$ than at $50 \%$ repolarization. Although $\triangle \mathrm{APD}$ is very small for ranolazine, the larger extent of APD prolongations are shown for d,l-sotalol and dofetilide that are associated with the Class III action of blocking $I_{K r}$ which can lead to torsade de points. 


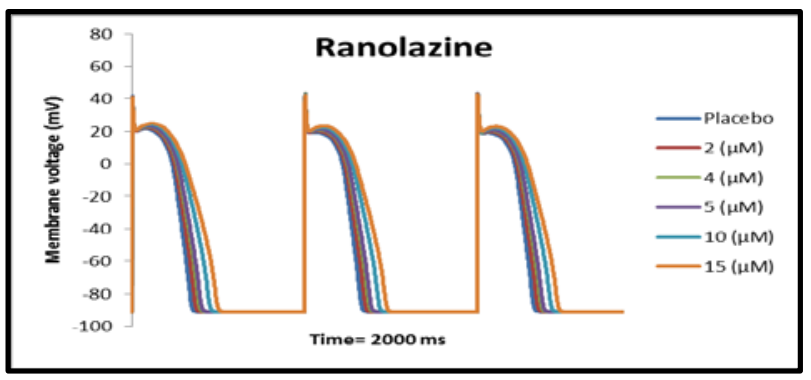

Figure 1. Concentration-dependent effects of ranolazine on cellular APs in the ORd endocardial human cell model.

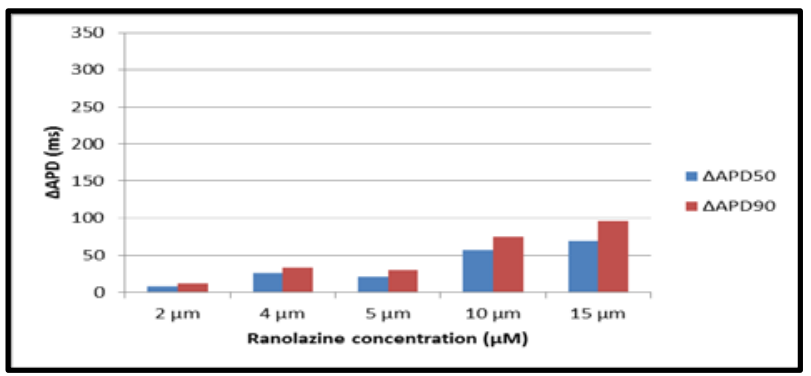

Figure 2. Concentration-dependent effects of ranolazine on $\triangle$ APD mutation in human ventricular myocyte. Adminstration of clinical range (ie, 2 to $6 \mu \mathrm{M}$ ) and high therapeutic ranolazine $(>10 \mu \mathrm{M})$ prolongs APD moderately.

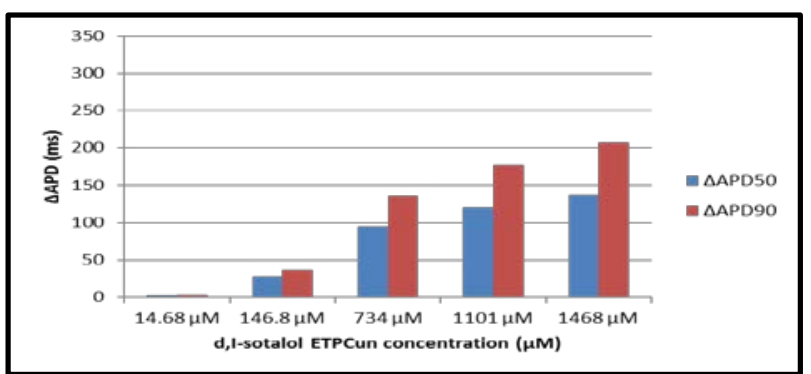

Figure 3. Concentration-dependent effects of d,l-sotalol, a selective $I_{K r}$ blocker, on $\triangle \mathrm{APD}$ mutation in human ventricular myocyte. Adminstration of d,l-sotalol produces a proportional increase in APD.

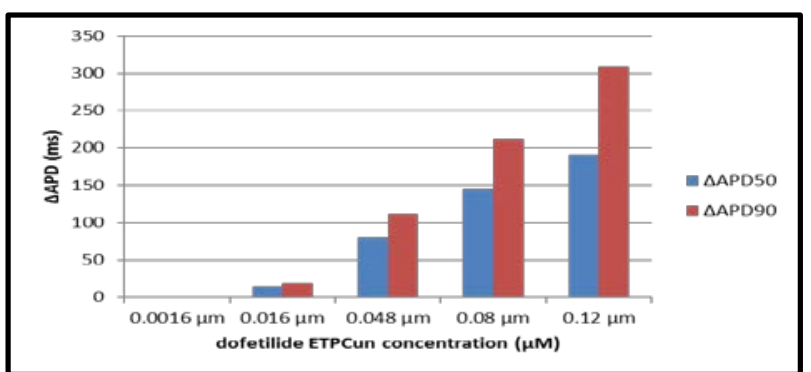

Figure 4. Concentration-dependent effects of dofetilide on $\triangle \mathrm{APD}$ mutation in human ventricular myocyte. Dofetilide shows significant increase in APD for the concentration range $(0.048$ to $0.12 \mu \mathrm{M})$.

\subsection{Drug-drug interactions}

The simulations were performed for a fixed concentration of the ranolazine $(15 \mu \mathrm{M})$ with a combination of dofetilide and d,l-sotalol to evaluate experimental and/or clinical measures of electrophysiological effect on ventricular myocytes. The effect of ranolazine is shown at different concentrations of two selective $I_{K r}$ blocker compounds (Figures 5 and 6). Despite primary prolongation of APD of the in-vitromodelled electrophysiology on both drugs, ranolazine shortened the APD, leading to suppression of the dofetilide and d,l-sotalol for the initiation/maintenance of ventricular arrhythmias.

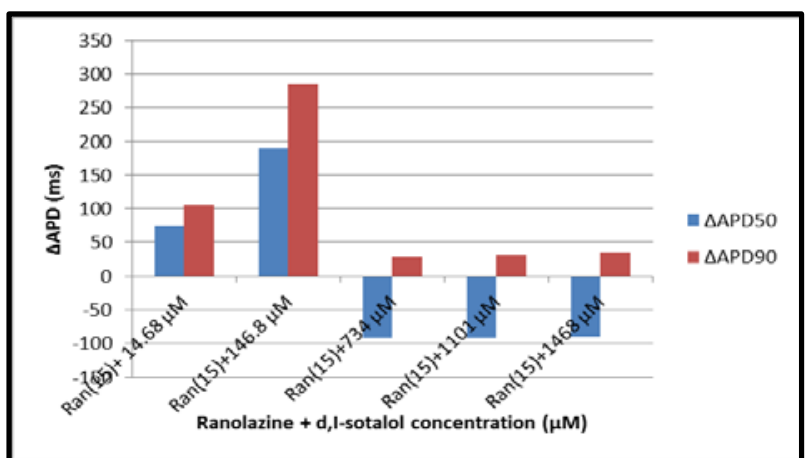

Figure 5. Impact of ranolazine (at $15 \mu \mathrm{M}$ ), with the combination of different d,l-sotalol plasma concentrations, on $\triangle \mathrm{APD}$ mutation $\left(\triangle A P D_{50}\right.$ and $\left.\triangle A P D_{90}\right)$ in human ventricular myocyte. Ranolazine (15 $\mu \mathrm{M})$ abbreviates the prolonged ventricular endocardial APD which is produced by d,1-sotalol at a concentration of $(\geq 734 \mu \mathrm{M})$.

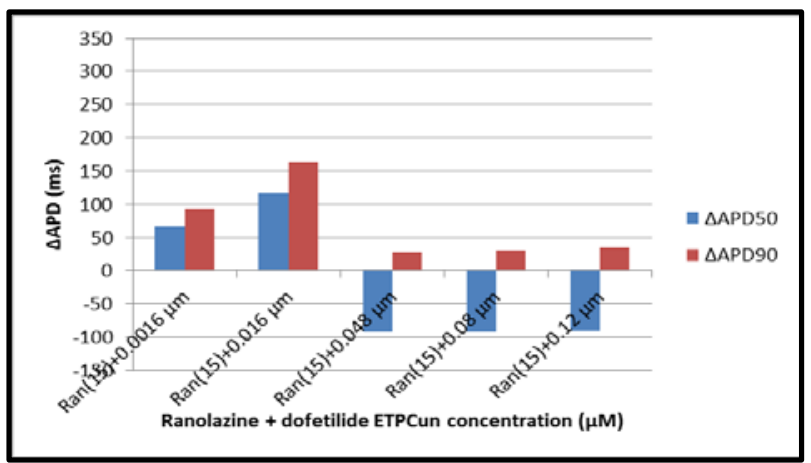

Figure 6. Impact of ranolazine (at $15 \mu \mathrm{M}$ ), with combination of different dofetilide plasma concentrations, on $\triangle \mathrm{APD}$ mutation $\left(\triangle A P D_{50}\right.$ and $\left.\triangle A P D_{90}\right)$ in human ventricular myocyte. Ranolazine $(15 \mu \mathrm{M})$ abbreviates the prolonged ventricular endocardial APD which is produced by dofetilide at a concentration of $(\geq 0.048 \mu \mathrm{M})$. 


\section{Discussion and conclusions}

This study determined the impact of the antiarrhythmic effect of ranolazine on the cardiac cell electrophysiology mechanism and its interaction with proarrhythmic drugs. The aim was to investigate the electrophysiological effects of ranolazine at concentrations within the therapeutic range (i.e., 2 to $6 \mu \mathrm{M})$ and supertherapeutic ( $\geq$ $10 \mu \mathrm{M}$ ) including inhibition of $I_{\mathrm{NaL}}, I_{\mathrm{CaL}}$ and $I_{\mathrm{Kr}}$.

As shown in Figures 1 and 2, ranolazine prolongs APD modestly due to the Inhibition of $I_{K r}$. Although ranolazine slightly prolonged APD, it reduced the increase in APD induced by the selective $I_{K r}$ blockers d-sotalol and dofetalide in human cardiac ventricles (Figure 7), which demonstrates the pharmacological effect of ranolazine including inhibition of $I_{N a L}, I_{C a L}$ and $I_{K r}$ currents [4].

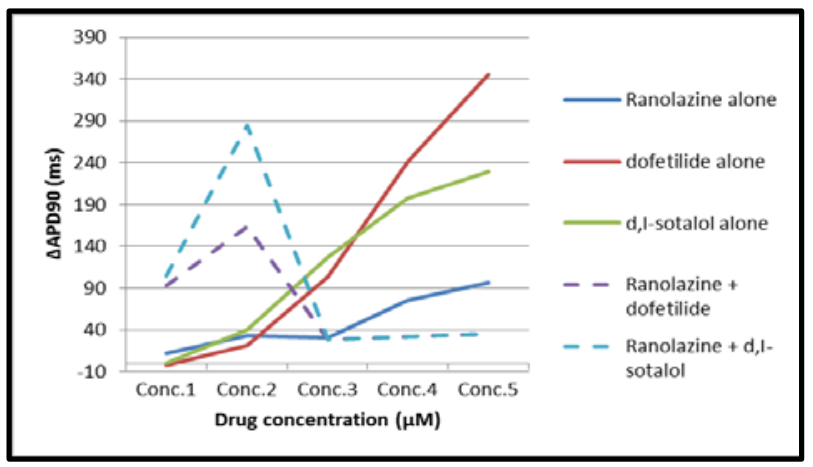

Figure 7. Summary of the electrophysiological effects of compounds on ventricular myocytes and their interactions with ranolazine. Note: Conc. represents drug plasma concentrations used individually or in combination with drugs (Concentration values are shown in Figures 2-6).

Our results support the major role of the inhibition of $I_{N a L}$ and $I_{K r}$ in modulation and the reduction of APD by ranolazine, despite a modest increase in the APD, based on data from animal ventricular myocytes. The dynamic behaviour and the response to simulated ventricular myocytes in these models were consistent with experimental observation $[4,9,11]$.

In our future work, we will investigate the underlying anti-arrhythmic action of ranolazine mechanisms including metabolic and antiischaemic influences in human ventricular moyocytes.

\section{Acknowledgements}

This study was supported by Simcyp (a Certara Company).

\section{References}

[1] Chaitman BR, Pepine CJ, Parker JO, Skopal J, Chumakova G, Kuch J, et al. Effects of ranolazine with atenolol, amlodipine, or diltiazem on exercise tolerance and angina frequency in patients with severe chronic angina: A randomized controlled trial. JAMA. 2004 Jan 21;291(3):309-16.

[2] Chaitman BR. Ranolazine for the treatment of chronic angina and potential use in other cardiovascular conditions. Circulation. 2006;113(20):2462-72.

[3] Song Y, Shryock JC, Wu L, Belardinelli L. Antagonism by ranolazine of the pro-arrhythmic effects of increasing late INa in guinea pig ventricular myocytes. $\mathrm{J}$ Cardiovasc Pharmacol. 2004;44(2):192-9.

[4] Antzelevitch C, Belardinelli L, Zygmunt AC, Burashnikov A, Di Diego JM, Fish JM, et al. Electrophysiological effects of ranolazine, a novel antianginal agent with antiarrhythmic properties. Circulation. 2004;110(8):904 10.

[5] Belardinelli L, Shryock JC, Fraser H. Inhibition of the late sodium current as a potential cardioprotective principle: effects of the late sodium current inhibitor ranolazine. Heart. 2006;92 Suppl 4:iv6-14.

[6] Reffelmann T, Kloner RA. Ranolazine: an anti-anginal drug with further therapeutic potential. Expert Rev Cardiovasc Ther. Taylor \& Francis; 2010 Mar 10;8(3):319-29.

[7] Tamargo J, Duarte J, Caballero R, Delpón E. New therapeutic targets for the development of positive inotropic agents. Discovery medicine. 2011. p. 381-92.

[8] O’Hara T, Virág L, Varró A, Rudy Y. Simulation of the undiseased human cardiac ventricular action potential: model formulation and experimental validation. PLoS Comput Biol. 2011 May;7(5):e1002061.

[9] Antzelevitch C, Burashnikov A, Sicouri S, Belardinelli L. Electrophysiological Basis for the Antiarrhythmic Actions of Ranolazine. October. 2011;141(4):520-9.

[10] Okada J -i., Yoshinaga T, Kurokawa J, Washio T, Furukawa T, Sawada K, et al. Screening system for druginduced arrhythmogenic risk combining a patch clamp and heart simulator. Sci Adv. 2015;1(4):e1400142-e1400142.

[11] Antoons G, Oros A, Beekman JDM, Engelen MA, Houtman MJC, Belardinelli L, et al. Late Na+ Current Inhibition by Ranolazine Reduces Torsades de Pointes in the Chronic Atrioventricular Block Dog Model. J Am Coll Cardiol. Elsevier Inc.; 2010;55(8):801-9.

[12] Polak S, Wiśniowska B, Fijorek K, Glinka A, Mendyk A. In vitro-in vivo extrapolation of drug-induced proarrhythmia predictions at the population level. Drug Discov Today. 2014;19:275-81.

Address for correspondence.

Mitra Abbasi

Simcyp Limited (a Certara Company)

Blades Enterprise Centre, John Street, Sheffield, S2 4SU, United Kingdom

Mitra.Abbasi@certara.com 